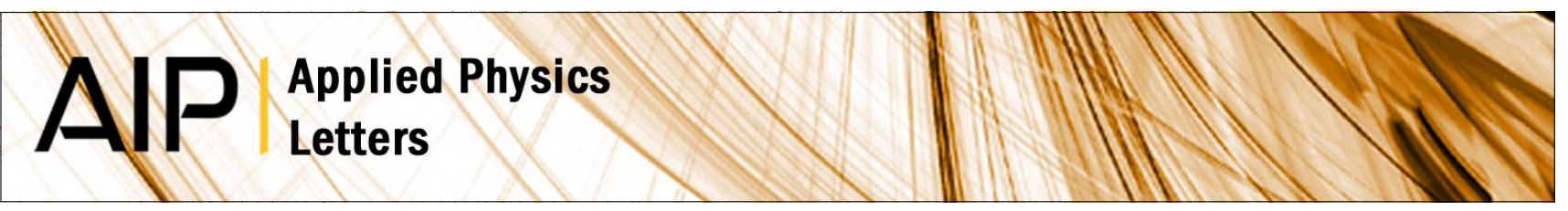

\title{
Probing buried layers by photoelectron spectromicroscopy with hard x-ray excitation
}

C. Wiemann, M. Patt, S. Cramm, M. Escher, M. Merkel et al.

Citation: Appl. Phys. Lett. 100, 223106 (2012); doi: 10.1063/1.4722940

View online: http://dx.doi.org/10.1063/1.4722940

View Table of Contents: http://apl.aip.org/resource/1/APPLAB/v100/i22

Published by the American Institute of Physics.

\section{Related Articles}

Largely enhanced photocurrent via gap-mode plasmon resonance by a nanocomposite layer of silver nanoparticles and porphyrin derivatives fabricated on an electrode

Appl. Phys. Lett. 101, 063103 (2012)

Valence band offset of $\mathrm{n}-\mathrm{ZnO} / \mathrm{p}-\mathrm{MgxNi1}-\mathrm{xO}$ heterojunction measured by $\mathrm{x}$-ray photoelectron spectroscopy Appl. Phys. Lett. 101, 052109 (2012)

Interfacial electronic properties of the heterojunctions $\mathrm{C} 60 /$ rubrene/Au and rubrene/C60/Au

J. Appl. Phys. 112, 023711 (2012)

Band offset measurements of the GaN/dielectric interfaces

J. Appl. Phys. 112, 024508 (2012)

Microwave absorption in nanocomposite material of magnetically functionalized carbon nanotubes

J. Appl. Phys. 112, 024302 (2012)

\section{Additional information on Appl. Phys. Lett.}

Journal Homepage: http://apl.aip.org/

Journal Information: http://apl.aip.org/about/about_the_journal

Top downloads: http://apl.aip.org/features/most_downloaded

Information for Authors: http://apl.aip.org/authors

\section{ADVERTISEMENT}

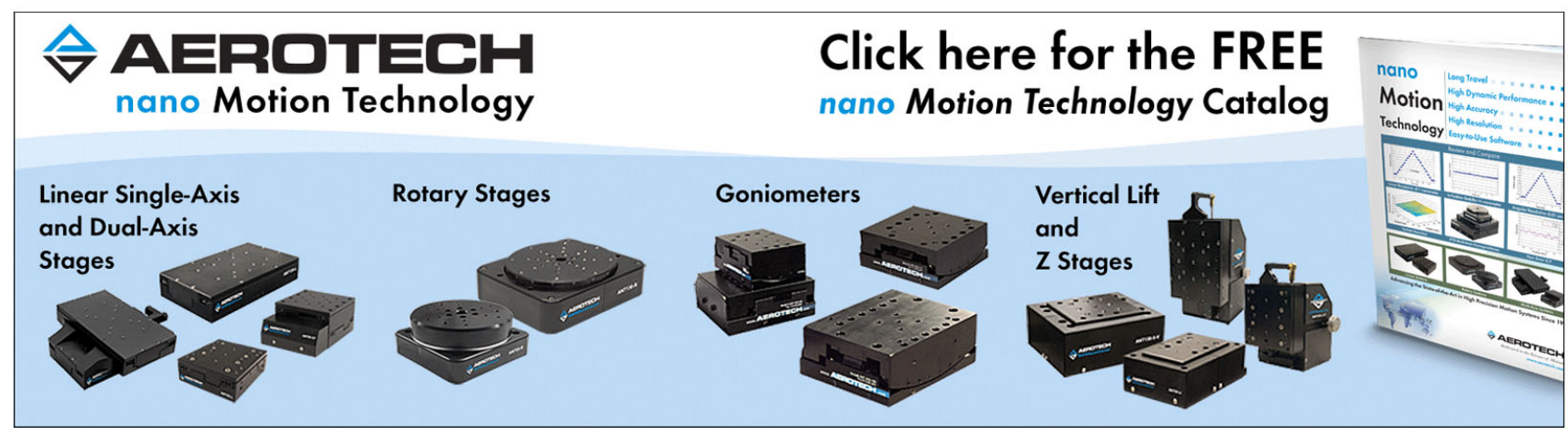




\title{
Probing buried layers by photoelectron spectromicroscopy with hard x-ray excitation
}

\author{
C. Wiemann, ${ }^{1}$ M. Patt, ${ }^{1}$ S. Cramm, ${ }^{1}$ M. Escher,${ }^{2}$ M. Merkel,${ }^{2}$ A. Gloskovskii, ${ }^{3}$ S. Thiess, ${ }^{4}$ \\ W. Drube ${ }^{4}$ and C. M. Schneider ${ }^{1,5}$ \\ ${ }^{1}$ Peter Grünberg Institute (PGI-6) and JARA-FIT, Research Centre Jülich, D-52425 Jülich, Germany \\ ${ }^{2}$ FOCUS GmbH, D-65510 Hünstetten, Germany \\ ${ }^{3}$ Institut für Anorganische Chemie und Analytische Chemie, Johannes Gutenberg-Universität Mainz, \\ D-55128 Mainz, Germany \\ ${ }^{4}$ DESY Photon Science, Deutsches Elektronen-Synchrotron, D-22603 Hamburg, Germany \\ ${ }^{5}$ Fakultät f. Physik and Center for Nanointegration Duisburg-Essen (CENIDE), Universität Duisburg-Essen, \\ D-47048 Duisburg, Germany
}

(Received 21 March 2012; accepted 14 May 2012; published online 30 May 2012)

\begin{abstract}
We report about a proof-of-principle experiment which explores the perspectives of performing hard x-ray photoemission spectromicroscopy with high lateral resolution. Our results obtained with an energy-filtered photoemission microscope at the PETRA III storage ring facility using hard $\mathrm{x}$-ray excitation up to $6.5 \mathrm{keV}$ photon energy demonstrate that it is possible to obtain selected-area $\mathrm{X}$-ray photoemission spectra from regions less than $500 \mathrm{~nm}$ in diameter. (C) 2012 American Institute of Physics. [http://dx.doi.org/10.1063/1.4722940]
\end{abstract}

Photoemission electron spectroscopy is a wellestablished method in surface physics and materials science. It is the tool of choice if it comes to a detailed analysis of the electronic and chemical states in solid state samples. ${ }^{1}$ Recent years have seen the evolution of photoelectron spectroscopy along various lines, aiming at an improvement of energy, lateral, time or spin resolution, an enhancement of the information depth, or the access to structural aspects. These evolution directions have been driven by specific requirements in solid state physics and materials research. The need to understand the microscopic nature of strongly correlated electron systems, for instance, has led to photoelectron spectroscopy with very high energy resolution. ${ }^{2}$ Likewise, the desire to study the electronic structure of novel magnetic materials in detail triggered the development of more efficient spin polarization detectors. ${ }^{3-5}$ The geometric arrangement of atoms at and adsorbates on surfaces can be conveniently deduced from photoelectron diffraction, i.e., the scattering of the outgoing photoelectron wave at neighboring atoms. ${ }^{6-8}$ The progress in laser technology nowadays enables pump-probe photoemission experiments with time resolution even in the femtosecond regime, which provide information about electronic excitations and dynamics as well as chemical reaction kinetics. ${ }^{9}$

The evolution of nanoscience with continuously decreasing feature size has created a strong need for electron spectroscopic information from sub- $\mu \mathrm{m}$ areas, i.e., spectromicroscopy or spectronanoscopy. This information is nowadays often provided by energy-filtering photoemission electron microscopes (PEEMs) with high lateral resolution which represent another line of development. ${ }^{10,11}$ Still, photoelectrons in a conventional photoemission experiment originate from the surface-near region, due to the kinetic energy dependence of the inelastic electron mean free path.

One way to enhance the information depth is by increasing the photoelectron kinetic energy using the excitation with hard $\mathrm{x}$-ray photons. The technique of hard x-ray photoemission spectroscopy (HAXPES) is the most recent development in the field. ${ }^{8,12-15}$ By using appropriate electron spectrometers an energy resolution down to about $70 \mathrm{meV}$ (Refs. 16 and 17) can be currently achieved. In order to make the advantages of HAXPES available to nanoscience, two approaches may be employed. First, a scanning type photoelectron spectroscopy which requires a sub-micrometer spot of the hard x-ray beam. Respective efforts in x-ray focusing are underway. ${ }^{18}$ Using post-focusing by, e.g., Kirkpatrick-Baez (KB) mirrors or compact refractive lenses (CRLs), spot sizes below $10 \times 10 \mu \mathrm{m}^{2}$ can be reached.

The second approach concerns energy-filtered fullfield photoemission microscopy using high kinetic energy photoelectrons. In this contribution we demonstrate in a proof-of-principle experiment that HAXPES with high lateral resolution is indeed feasible in an immersion lens microscope. At a photoelectron kinetic energy of $4.5 \mathrm{keV}$, we are still able to observe a clear core level photoemission signal from $\mathrm{SrTiO}_{3}$ through a $7 \mathrm{~nm}$ thick Au overlayer. The lateral resolution of this hard $\mathrm{x}$-ray photoemission electron microscopy (HAXPEEM) achieved so far is better than $500 \mathrm{~nm}$.

The lateral resolution is mainly determined by the electron-optical properties of the immersion lens system. This approach has not yet been explored in the context of HAXPES. The main reason may be a low signal level expected due to the unfavorable combination of low photoexcitation cross sections in the hard $\mathrm{x}$-ray regime and low transmission of immersion lens systems at high kinetic electron energies.

We note that PEEM has been used in combination with hard x-rays as a spatially resolving total electron yield detector. Here, spectroscopic information can be obtained in an x-ray absorption setup (NEXAFS), while the low-energy secondary electrons generated by the absorption process cascade are used for imaging. ${ }^{19-21}$

The working principle of immersion lens microscopes requires a large electric field of about $10 \mathrm{kV} / \mathrm{mm}$ to be 
established between the sample and the extractor lens. For brevity, we point out only one salient feature of such a microscope (for further details, see Refs. 22-24). By their lens design, the instruments fall into two classes, i.e., electromagnetic and electrostatic electron optical systems. Electromagnetic lenses require the sample to sit on high acceleration voltage, usually $\mathrm{V}_{\mathrm{ac}}=20 \mathrm{kV}$. In an electrostatic microscope column, however, an "inverted" potential scheme with the sample held close to ground and the extractor lens being put on high voltage applies. We will focus on this scheme which is advantageous for the energy-filtered mode. The photoexcitation releases photoelectrons with a spectrum of kinetic energies $\Delta \mathrm{E}_{\mathrm{k}}$ which are accelerated into the immersion lens. After forming the image behind the objective lens system, the electrons are retarded to the column potential $\left(\mathrm{V}_{\mathrm{C}}=1 \mathrm{kV}\right)$ and in the total yield mode pass several projection lens stages which subsequently magnify the image onto the detector. Note that in the column the electrons are moving with a spread of drift energies $\Delta \mathrm{E}_{\mathrm{D}}=\mathrm{eV}_{\mathrm{C}}$ $-\mathrm{eV}_{\mathrm{S}}+\Delta \mathrm{E}_{\mathrm{k}}$ (sample potential $\mathrm{V}_{\mathrm{S}}=0 \mathrm{~V}$ in total yield operation).

For our studies we moved the NanoESCA instrument normally operated at the Elettra NanoSpectroscopy beamline $^{25}$ to the PETRA III storage ring facility (DESY, Hamburg). In the energy-filtered mode a double-hemispherical analyzer energy selects the electrons in between the projection stages. ${ }^{26}$ The transmission of the microscope decreases with photoelectron kinetic energy as $1 / E_{k}$ to about $0.15 \%$ at $6.5 \mathrm{keV}$ using a $500 \mu \mathrm{m}$ diameter aperture. The retardation towards the pass energy of the analyzer $\left(E_{P}=100 \mathrm{eV}\right)$ and the passage of the double hemispheres introduces no further reduction of the available phase space besides the energy filtering. As the analyzer is operated at fixed pass energy, the photoelectron spectrum is moved across the entrance slit of the analyzer by scanning the sample potential $\mathrm{V}_{\mathrm{S}}$. Since we used a maximum photon energy of $6.5 \mathrm{keV}$ in our HAXPEEM experiment, this resulted in $\mathrm{V}_{\mathrm{S}} \leq 6.5 \mathrm{kV}$ requiring sufficient sample insulation. In order to preserve the image quality in the microscope column over a broad kinetic energy range we increased the values for $\mathrm{V}_{\mathrm{ac}}$ and $\mathrm{V}_{\mathrm{C}}$ to $+24 \mathrm{kV}$ and $+2 \mathrm{kV}$, respectively.

The experiments were performed on x-ray undulator beamline P09 at PETRA III, providing photon energies between 3 and $15 \mathrm{keV}$ for HAXPES experiments. ${ }^{27}$ For the experiments photon energies of 4500 and $6500 \mathrm{eV}$ were selected using the primary high heat load $\mathrm{Si}(111)$ monochromator yielding an energy band pass of $0.85 \mathrm{eV}$. The microscope's electron optical axis was oriented along the vertical direction at an angle of $75^{\circ}$ with the incoming synchrotron radiation beam. The measurements were carried out with linearly p-polarized light impinging on the sample surface at an angle of $15^{\circ}$. The images were recorded with a slow-scan 14 bit CCD camera.

For magnification calibration and resolution testing we used a checkerboard-patterned Au/Si sample (Plano GmbH). The structure comprises of several interlaced square patterns with side lengths of 1,10 , and $100 \mu \mathrm{m}$, respectively. The energy-filtered image shown in Fig. 1 was recorded on the Au $3 \mathrm{~d}_{5 / 2}$ core level emission line with an $\mathrm{x}$-ray excitation of $\mathrm{h} \nu=6.5 \mathrm{keV}$. This corresponds to imaging at a kinetic

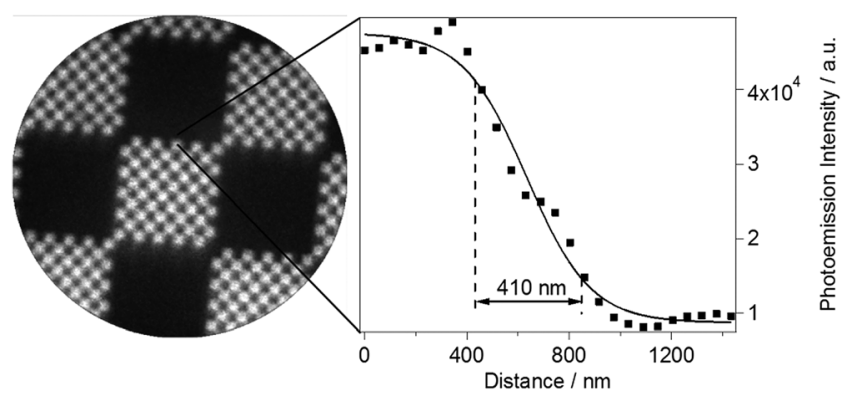

FIG. 1. Au/Si calibration sample imaged on the $\mathrm{Au} 3 \mathrm{~d}_{5 / 2}$ photoemission line at a kinetic energy of $4294 \mathrm{eV}$ (left). Line scan across a square edge and $16 / 84 \%$ profile fit (right).

energy of $4294 \mathrm{eV}$. The line scan across the Au square edge demonstrates a spatial resolution $\Delta x=410 \mathrm{~nm}$. Since the image was taken with a large diameter contrast aperture $(500 \mu \mathrm{m})$ to allow for moderate exposition times at the rather low count rates, this is in line with the theoretical expectation. The usage of long exposition time (total $120 \mathrm{~min}$ ) also shows the excellent long time stability of the instrument on this length scale.

In order to demonstrate the feasibility of imaging spectroscopy with a photoelectron signal from buried layers, we used a different sample, which consisted of a $\mathrm{SrTiO}_{3}$ (STO) thin film grown by pulsed laser deposition. It was capped by a $7 \mathrm{~nm}$ thick Au overlayer which was subsequently patterned into an array of $10 \times 10 \mu \mathrm{m}^{2}$ square electrodes by optical lithography and wet chemical etching. Such samples are extensively used in resistive switching experiments. ${ }^{28}$ The sample was imaged in the microscope without further surface treatment.

Figure 2(a) shows a PEEM image taken in threshold photoemission at low kinetic energy. The field of view is approximately $40 \mu \mathrm{m}$ and contains three Au electrode pads measuring $10 \times 10 \mu \mathrm{m}^{2}$ each. The rightmost pad shows surface damage due to electrical treatment in preceding switching experiments. Figure 2(b) shows the same area of the sample surface, this time imaged at a kinetic energy of $4560 \mathrm{eV}$ corresponding to the $\mathrm{Sr} 2 \mathrm{p}_{3 / 2}$ level at an excitation energy of $\mathrm{h} \nu=6500 \mathrm{eV}$. The image has been corrected for detector dark counts and divided by a bright field image to reduce detector inhomogeneities. Here, the Au pads appear dark, because the $\mathrm{Sr} 2 \mathrm{p}$ emission from the underlying STO layers is attenuated in the $7 \mathrm{~nm}$ top layer. On the rightmost
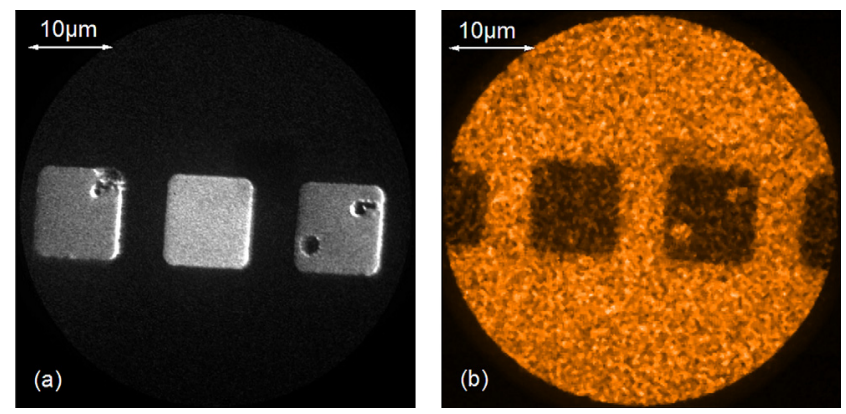

FIG. 2. Comparison of imaging at low and high kinetic energies. (a) Image at low kinetic energy (threshold photoemission using mercury discharge lamp). The field of view is $40 \mu \mathrm{m}$. (b) Image of the same region of the sample with $\mathrm{Sr} 2 \mathrm{p}_{3 / 2}$ photoelectrons (kinetic energy $4560 \mathrm{eV}$ ) excited with hard $\mathrm{x}$-rays of $\mathrm{h} \nu=6500 \mathrm{eV}$. 


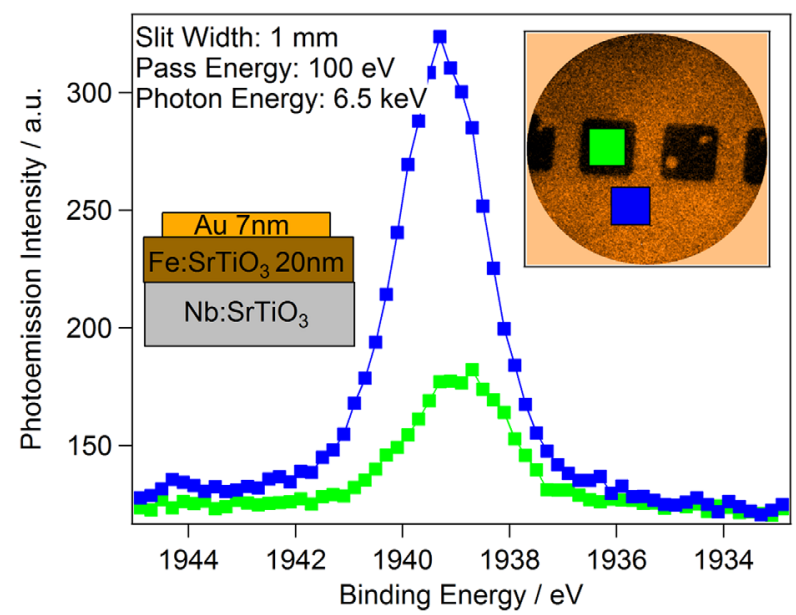

FIG. 3. Selected area hard X-ray photoemission spectra from Au/STO. Inset top right: definition of regions of interest. Inset left: sample layer composition.

square the damaged areas exhibit a brighter contrast, indicating that the damage is related to a local removal of gold from the STO surface.

In order to obtain detailed HAXPES information on the sample we recorded a stack of images as function of kinetic energy scanning across characteristic photoemission lines. The example shown in Fig. 3 maps the spectral signatures of the $\mathrm{Sr} 2 \mathrm{p}_{3 / 2}$ core level photoemission at different regions of interest (RoI) on the sample surface. The spectra are calculated within the RoIs marked in the inset, each of them corresponding to surface areas of approximately $8 \times 8 \mu \mathrm{m}^{2}$ size. This approach yields representative $\mathrm{Sr} 2 \mathrm{p}_{3 / 2}$ core level spectra for the Au covered and uncovered surface. The intensity loss by a factor of 3.4 between the covered and uncovered regions leads to a value of $8.5 \mathrm{~nm}$ for the electron inelastic mean free path (IMFP), which compares reasonably well with the theoretical predictions for the IMFP of $4.4 \mathrm{~nm}$ at a kinetic energy of $4560 \mathrm{eV} .^{29}$ The deviation may be assigned to surface roughness in the Au layer. We also note a small difference in the binding energy position of the photoemission peak maxima. The photoemission peak from the uncovered surface is found at $0.4 \mathrm{eV}$ higher binding energy compared to the signal from the Au covered area. We would like to recall that on the uncovered surface we basically probe the bulk of STO, whereas the Sr photoemission signal from underneath the $\mathrm{Au}$ electrodes stems mainly from the interfacial region. This result is a first indication that the interfacial states at the metal electrode differ from the bulk electronic states. Due to the local nature of the spectroscopy we can really pinpoint this difference, the origin of which will be subject to future studies.

In conclusion, we have presented proof-of-principle data that demonstrates the feasibility of operating an energyfiltered PEEM at the high kinetic energies present in hard $\mathrm{X}$-ray photoemission. The combination of parallel imaging at sub-micrometer spatial resolution with the high probing depth of HAXPES is a promising technique in the context of structured layered system as are commonly encountered in device engineering.

Thanks are due to $\mathrm{Ch}$. Lenser for providing the STO sample. Financial support through the Deutsche Forschungsgemeinschaft within Sonderforschungsbereich 917 "Nanoswitches" is gratefully acknowledged.

${ }^{1}$ S. Hüfner, Photoemission Spectroscopy, Springer Series in Solid State Physics (Springer, Berlin, 1995), Vol. 82.

${ }^{2}$ S. Hüfner, Very High Resolution Photoelectron Spectroscopy, Lecture Notes in Physics (Springer, Berlin, 2007).

${ }^{3}$ F. U. Hillebrecht, R. M. Jungblut, L. Wiebusch, C. Roth, H. B. Rose, D. Knabben, C. Bethke, N. B. Weber, S. Manderla, U. Rosowski, and E. Kisker, Rev. Sci. Instrum. 73, 1229 (2002).

${ }^{4}$ A. Winkelmann, D. Hartung, H. Engelhard, C. T. Chiang, and J. Kirschner, Rev. Sci. Instrum. 79, 83303 (2008).

${ }^{5}$ M. Kolbe, P. Lushchyk, B. Petereit, H. J. Elmers, G. Schönhense, A. Oelsner, C. Tusche, and J. Kirschner, Phys. Rev. Lett. 107, 207601 (2011).

${ }^{6}$ C. Westphal, Surf. Sci. Rep. 50, 1 (2003).

${ }^{7}$ D. P. Woodruff, Surf. Sci. Rep. 62, 1 (2007).

${ }^{8}$ C. Fadley, J. Electron Spectrosc. Relat. Phenom. 178-179, 2 (2010).

${ }^{9}$ U. Bovensiepen, H. Petek, and M. Wolf, Dynamics at Solid State Surfaces and Interfaces (Wiley-VCH, Berlin, 2012).

${ }^{10}$ E. Bauer, J. Electron Spectrosc. 114-116, 975 (2001).

${ }^{11}$ M. Escher, N. Weber, M. Merkel, B. Krömker, D. Funnemann, S. Schmidt, F. Reinert, F. Forster, S. Hüfner, P. Bernhard, C. Ziethen, H. J. Elmers, and G. Schönhense, J. Electron Spectrosc. 144-147, 1179 (2005).

${ }^{12}$ A. Sekiyama, H. Fujiwara, S. Imada, S. Suga, H. Eisaki, S. I. Uchida, K. Takegahara, H. Harima, Y. Saitoh, I. A. Nekrasov, G. Keller, D. E. Kondakov, A. V. Kozhevnikov, T. Pruschke, K. Held, D. Vollhardt, and V. I. Anisimov, Phys. Rev. Lett. 93, 156402 (2004).

${ }^{13}$ W. Drube, Nucl. Instrum. Meth. A 547, 87 (2005).

${ }^{14}$ K. Kobayashi, Nucl. Instrum. Meth. A 601, 32 (2009).

${ }^{15}$ C. S. Fadley, Nucl. Instrum. Meth. A 601, 8 (2009).

${ }^{16}$ G. Panaccione, G. Cautero, A. Fondacaro, M. Grioni, P. Lacovig, G. Monaco, F. Offi, G. Paolicelli, M. Sacchi, G. Stefani, and P. Torelli, Nucl. Instrum. Meth. A 547, 56 (2005).

${ }^{17}$ Y. Takata, M. Yabashi, K. Tamasaku, Y. Nishino, D. Miwa, T. Ishikawa, E. Ikenaga, K. Horiba, S. Shin, M. Arita, K. Shimada, H. Namatame, M. Taniguchi, H. Nohira, T. Hattori, S. Södergren, B. Wannberg, and K. Kobayashi, Nucl. Instrum. Meth. A 547, 50 (2005).

${ }^{18}$ H. Mimura, S. Matsuyama, H. Yumoto, H. Hara, K. Yamamura, Y. Sano, M. Shibahara, K. Endo, Y. Mori, Y. Nishino, K. Tamasaku, M. Yabashi, T. Ishikawa, and K. Yamauchi, Jpn. J. Appl. Phys. 44, L539 (2005).

${ }^{19}$ Y. Hwu, W. Tsai, L. Chang, C. Chen, C. Wu, D. Noh, J. Je, G. Fecher, M. Bertolo, G. Berger, and G. Margaritondo, Jpn. J. Appl. Phys. 38-1, 646 (1999). Available at: http://jjap.jsap.jp/link?JJAPS/38S1/646/.

${ }^{20}$ Y. Hwu, W. Tsai, B. Lai, J. Je, G. Fecher, M. Bertolo, and G. Margaritondo, Surf. Sci. 480, 188 (2001).

${ }^{21}$ T. Taniuchi, T. Wakita, M. Takagaki, N. Kawamura, M. Suzuki, T. Nakamura, K. Kobayashi, M. Kotsugi, M. Oshima, H. Akinaga, H. Muraoka, and K. Ono, AIP Conf. Proc. 879, 1353 (2007).

${ }^{22}$ G. Schönhense, J. Phys. - Condens. Matter 11, 9517 (1999).

${ }^{23}$ E. Bauer, J. Phys. - Condens. Matter 13, 11391 (2001).

${ }^{24}$ R. M. Tromp, J. B. Hannon, A. W. Ellis, W. Wan, A. Berghaus, and O. Schaff, Ultramicroscopy 110, 852 (2010).

${ }^{25}$ C. Wiemann, M. Patt, I. P. Krug, N. B. Weber, M. Escher, M. Merkel, and C. M. Schneider, e-J. Surf. Sci. Nanotechnol. 9, 395 (2011).

${ }^{26}$ M. Escher, N. Weber, M. Merkel, C. Ziethen, P. Bernhard, G. Schönhense, S. Schmidt, F. Förster, F. Reinert, B. Krömker, and D. Funnemann, J. Phys. - Condens. Matter. 17, S1329 (2005).

${ }^{27}$ A. Gloskovskii, G. Stryganyuk, G. H. Fecher, C. Felser, S. Thiess, H. Schulz-Ritter, W. Drube, G. Berner, M. Sing, R. Claessen, and M. Yamamoto, J. Electron Spectrosc. 185, 47 (2012).

${ }^{28}$ R. Waser, R. Dittmann, G. Staikov, and C. Szot, Adv. Mater. 21, 1 (2009).

${ }^{29}$ S. Tanuma, C. J. Powell, and D. R. Penn, Surf. Interface Anal. 43, 689 (2011). 